Bull. Austral. Math. Soc.

VoL. 39 (1989) [1-9]

\title{
ANOTHER APPROACH TO EXTENSIONS OF CONTINUOUS MAPPINGS
}

\author{
SHU-HaO SUN
}

\begin{abstract}
First, we give a more general extension theorem about continuous mappings. It is shown that the Taimanov-Eilenberg-Steenrod extension theorem and the Engelking extension theorem are special cases and that this theorem implies the Dugunji extension theoren. The localic version of this theorem also generalises Joyal's extension theorem for locales. Then, using the same technique, we obtain another more interesting extension theorem and its applications. In particular, we sharpen a well-known result due to Wallman.
\end{abstract}

Throughout this paper, all notation is standard. Undefined notions may be found in [3] and [7] (topological notions in [3] and lattice-theoretic notions in [7]).

To determine a continuous extension, a standard method is to use the convergence of a filter or a net. All the proofs in 1, 2, 3, 4,6 depend on this method. However, we provide another technique here, which seems to be new, to construct an extension of a continuous mapping. This technique can be used in cases where the image space is not the Hausdorff. Our second main result (Theorem 4) will illustrate the point. As an interesting application of the theorem, we shall construct well-compactifications for any $T_{0}$-spaces such that this well-compactification has a weaker universal property similar to that possessed by Stone-C̆ech compactifications. In particular, we shall sharpen a well-known result due to Wallman.

The following theorem is our first main result.

Theorem 1. Let $A$ be a dense subspace of a topological space $X$, and $f$ a continuous mapping of $A$ to a $T_{3}$-space $Y$. The mapping $f$ has a continuous extension over $X$ if and only if for every open cover $\left\{V_{\alpha}: \alpha \in \Lambda\right\}$ of $Y$, there exists an open cover $\left\{U_{i}: i \in I\right\}$ of $X$ such that the open cover $\left\{U_{i} \cap A: i \in I\right\}$ of the subspace $A$ is a refinement of $\left\{f^{-1}\left(V_{\alpha}\right): \alpha \in \Lambda\right\}$.

Proof: The necessity is obvious. To show the converse, first note that the assumed condition is equivalent to the condition that $\left\{\operatorname{Exf}^{-1}\left(V_{\alpha}\right): \alpha \in \Lambda\right\}$ is an open cover of $X$, where $\operatorname{ExD}=X \backslash \overline{(A \backslash D)}$. Now let lattices of the open sets of $Z$ be denoted by $O(Z)$. Since $f$ is continuous, we have a frame mapping $f^{-1}: O(Y) \rightarrow O(A)$, that is $f^{-1}$ preserves finite meets and arbitrary joins. Now note that $O(A)$ is isomorphic to

Received 6 December 1987

Copyright Clearance Centre, Inc. Serial-fee code: 0004-9729/89 \$A2.00+0.00. 
the set $\{\operatorname{ExU}: U \in O(A)\}$ as a meet-sublattice of $O(X)$, that is $\operatorname{Ex}: O(A) \rightarrow O(X)$ is injective and preserves finite meets. Thus we define a mapping $g^{*}: O(Y) \rightarrow O(X)$ as follows:

$$
g^{*}(V)=\bigcup\left\{\operatorname{Exf}^{-1}\left(V^{\prime}\right): \bar{V}^{\prime} \subseteq V\right\}, \text { for each } V \in O(Y)
$$

Clearly, we have $g^{*}(\emptyset)=\emptyset$ and $g^{*}(Y)=X$ by the regularity.

Now we want to show that $g^{*}$ preserves finite meets and arbitrary joins. Let $V_{1}, V_{2} \in O(Y)$, then

$$
\begin{aligned}
g^{*}\left(V_{1}\right) \cap g^{*}\left(V_{2}\right) & =\left(\bigcup\left\{\operatorname{Exf}^{-1}\left(V^{\prime}\right): \vec{V}^{\prime} \subseteq V_{1}\right\}\right) \cap\left(\bigcup\left\{\operatorname{Exf}^{-1}\left(V^{\prime \prime \prime}\right): \bar{V}^{\prime \prime} \subseteq V_{2}\right\}\right) \\
& =\bigcup\left\{\operatorname{Exf}^{-1}\left(V^{\prime}\right) \cap \operatorname{Exf}^{-1}\left(V^{\prime \prime}\right): \bar{V}^{\prime} \subseteq V_{1}, \bar{V}^{\prime \prime} \subseteq V_{2}\right\} \\
& =\bigcup\left\{\operatorname{Exf}^{-1}(W): \bar{W} \subseteq V_{1} \cap V_{2}\right\}=g^{*}\left(V_{1} \cap V_{2}\right) .
\end{aligned}
$$

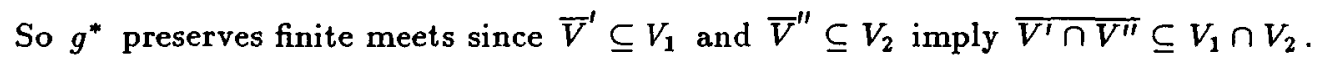

To show $g^{*}\left(\bigcup_{\alpha \in \Lambda} V_{\alpha}\right)=\bigcup_{\alpha \in \Lambda} f^{*}\left(V_{\alpha}\right)$, we consider the union $\bigcup_{\alpha \in \Lambda} V_{\alpha}$ in $O(Y)$; by regularity, we also have $\bigcup_{\alpha \in \Lambda} V_{\alpha}=\bigcup T$, where $T=\left\{W_{i} \in O(Y): \bar{W}_{i} \subseteq\right.$ $V_{\alpha_{i}}$ for some $\left.\alpha_{i} \in \Lambda\right\}$. Thus we have $\operatorname{Exf}^{-1}\left(W_{i}\right) \subseteq g^{*}\left(V_{\alpha_{i}}\right)$ for each $W_{i} \in T$.

If $\bar{V}^{\prime} \subseteq \bigcup_{\alpha \in \Lambda} V_{\alpha}=\bigcup T$, that is, $\left(Y \backslash \bar{V}^{\prime}\right) \cup\left(\bigcup W_{i}\right)=Y$, then, by the assumed condition, we have $\bigcup\left\{\operatorname{Exf}^{-1}(W): W \in T\right\} \cup\left(\operatorname{Exf}^{-1}\left(Y \backslash \bar{V}^{\prime}\right)\right)=X$, hence $\operatorname{Exf}^{-1}\left(V^{\prime}\right) \subseteq \bigcup\left\{\operatorname{Exf}^{-1}(W): W \in T\right\} \bigcup\left(\operatorname{Exf}^{-1}\left(Y \backslash \bar{V}^{\prime}\right)\right)$. Since $\operatorname{Exf}^{-1}\left(V^{\prime}\right) \cap$ $\operatorname{Exf}^{-}\left(Y \backslash \bar{V}^{\prime}\right) \neq \emptyset$, so we have $\operatorname{Exf}^{-}\left(V^{\prime}\right) \subseteq \bigcup\left\{\operatorname{Exf}^{-1}(W): W \in T\right\} \subseteq \bigcup_{\alpha \in \Lambda} g^{*}\left(V_{\alpha}\right)$, therefore

$$
g^{*}\left(\bigcup_{\alpha \in \Lambda} V_{\alpha}\right)=\bigcup\left\{\operatorname{Exf}^{-1}\left(V^{\prime}\right): \bar{V}^{\prime} \subseteq \bigcup_{\alpha \in \Lambda} V_{\alpha}\right\} \subseteq \bigcup_{\alpha \in \Lambda} g^{*}\left(V_{\alpha}\right)
$$

But the reverse inequality is trivial since $g^{*}$ is order-preserving.

Now we can define $g_{*}: O(X) \rightarrow O(Y)$ as follows:

$$
g_{*}(U)=\bigcup\left\{V \in O(Y): g^{*}(V) \subseteq U\right\} \text { for each } U \in O(X) .
$$

Since $g^{*}$ preserves arbitrary joins, we have, for each pair $U \in O(X), V \in O(Y)$,

$$
V \subseteq g_{*}(U) \text { if and only if } g^{*}(V) \subseteq U
$$

Now we claim that for each $x \in X, g_{*}(X \backslash \overline{\{x\}})=Y \backslash\{y\}$ for some $y \in Y .\left(^{* *}\right)$. If $g_{*}(X \backslash \overline{\{x\}})=Y$ for some $x \in X$, by $\left(^{*}\right), g^{*}(Y) \subseteq X \backslash\{X\}$, but $g^{*}(Y)=X$, so we have a contradiction. 
If $g_{*}(X \backslash \overline{\{x\}})=Y \backslash F$, for some $x \in X$, where $F$ is not a single point set, let $y_{1}, y_{2} \in F y_{1} \neq y_{2}$. Then we have $V_{1}, V_{2} \in O(Y)$ such that $V_{1} \cap V_{2}=\emptyset$, and $x_{i} \in V_{i}, i=1,2$, hence $V_{1} \cap V_{2} \subseteq g_{*}(X \backslash \overline{\{x\}}) g^{*}\left(V_{1} \cap V_{2}\right) \subseteq X \backslash \overline{\{x\}}$, by finite meet-preservation $g^{*}\left(V_{1}\right) \cap g^{*}\left(V_{2}\right) \subseteq X \backslash \overline{\{x\}}$, so we have some $i \in\{1,2\}$, such that $g^{*}\left(V_{i}\right) \subseteq X \backslash \overline{\{x\}}, V_{i} \subseteq g_{*}(X \backslash \overline{\{x\}})=Y \backslash F$. But $x_{i} \in V_{i} \cap F$, so again we have a contradiction. Thus formula $\left({ }^{* *}\right)$ is established. By $\left({ }^{* *}\right)$, we can define a mapping $g: X \rightarrow Y$ as follows:

$$
\text { for each } x \in X, g(x)=y \text { if and only if } g_{*}(X \backslash \overline{\{x\}})=Y \backslash\{y\} \text {. }
$$

We claim that $g$ is a continuous extension of $f$.

To show that $g$ is continuous, it suffices to show $g^{-1}=g^{*}$. For each $V \in O(Y)$, we have $g^{-1}(V)=\{x \in X: g(x) \in V\}=\{x: V \nsubseteq Y \backslash\{g(x)\}\}=\left\{x: V \nsubseteq g_{*}(X \backslash \overline{\{x\}})\right\}=$ $\left\{x: g^{*}(V) \nsubseteq X \backslash \overline{\{x\}}\right\}=\left\{x: x \in g^{*}(V)\right\}=g^{*}(V) \in O(X)$.

To show that $g$ is an extension of $f$, we first show $g^{-1}(V) \cap A=f^{-1}(V)$ for each $V \in 0(Y)$. In fact, $g^{-1}(V) \cap A=g^{*}(V) \cap A=\left(\cup\left\{\operatorname{Exf}^{-1}\left(V^{\prime}\right): \bar{V}^{\prime} \subseteq V\right\}\right) \cap A=$ $\cup\left\{\operatorname{Exf}^{-1}\left(V^{\prime}\right) \cap A: \bar{V}^{\prime} \subseteq V\right\}=\cup\left\{f^{-1}\left(V^{\prime}\right): \bar{V}^{\prime} \subseteq V\right\}=f^{-1}(V)$. Now we can show that $g$ is as required. If $a \in A, f(a) \neq g(a)$, there exists a $V \in O(Y)$ such that $f(a) \in V$ and $g(a) \notin V$, but $a \in f^{-1}(V)=g^{-1}(V) \cap A$ implies $g(a) \in V$. It is a contradiction. This also completes the proof.

CorollarY 1. (Eilenberg-Steenrod-Taimanov [2, p.280]). Let $A$ be a dense subspace of a topological space and $f$ a continuous mapping of $A$ to a compact Hausdorff space $Y$. Then the mapping $f$ has a continuous extension over $X$ if and only if for each open cover $\left\{V_{i}\right\}_{i=1}^{k}$ of $Y$ there is an open cover $\left\{U_{i}\right\}_{i=1}^{m}$ of $X$ such that the open cover $\left\{U_{i} \cap A\right\}_{i-1}^{m}$ of $A$ is a refinement of $\left\{f^{-1}\left(V_{i}\right)\right\}_{i=1}^{k}$.

Corollary 2. (Englking-Vulih [4]). Let $A$ be a dense subspace of a topological space $X$ and $f$ a continuous mapping of $A$ to a real compact space $Y$. Then the mapping $f$ has a continuous extension over $X$ if and only if for every sequence $F_{1}, F_{2}, \ldots$ of closed subsets of $Y$ such that $\bigcap_{i=1} F_{i}=\emptyset$, we have $\bigcap_{i=1} \overline{f^{-1}\left(F_{i}\right)}=\emptyset$.

Proof: By Theorem 1, it is clear if $Y$ is a Lindelof space. Then by virtue of the lemma $([3, p .176])$, the proof is complete (note that a space is real compact if and only if it is homeomorphic to a closed subspace of a cartesian product of copies of the real line).

Corollary 3. (Dugundji). Let $D$ be a dense subspace of $X . Y$ a regular space and $f: D \rightarrow Y$ continuous. Then $f$ has a continuous extension $F: X \rightarrow Y$ if and only 
if the filterbase $f(D \cap \mathcal{U}(x))$ converges for each $x \in X$, where $\mathcal{U}(x)$ is the neighbourhood system of $x$. (see $[1, p .216])$

Proof: Let $\mathcal{V}$ be open cover of $Y$ such that $\bigcup\left\{\operatorname{Exf}^{-1}(V): V \in \mathcal{V}\right\} \neq X$. Then there is an $x \in X$ such that $x \notin \bigcup\left\{\operatorname{Exf}^{-1}(V): V \in \mathcal{V}\right\}$, that is, for each $U \in \mathcal{U}(x), V \in \mathcal{V}, U \nsubseteq \operatorname{Exf}^{-1}(V), U \cap D \nsubseteq f^{-1}(V)$, hence $f(U \cap D) \nsubseteq V$. But $f(D \cap \mathcal{U}(x))$ converges to some $y \in Y$; so there is a $V_{0} \in \mathcal{V}$ such that $y \in V_{0}$. Hence there exists an $U_{0} \in \mathcal{U}(x)$ such that $f\left(U_{0} \cap D\right) \subseteq V_{0}$. Thus we have a contradiction.

Mimicking the proof of Theorem 1, we have

Theonem 2. Let $A$ be a dense subspace of a topological space $X$ and $F$ be a continuous mapping of $A$ to a locally compact space $y$. The mapping $F$ has a continuous extension over $X$ if and only if there is an open cover $\left\{V_{\alpha}: \alpha \in \Lambda\right\}$ of $Y$ in which each element has a compact closure such that $\left\{\operatorname{Exf}^{-1}\left(V_{\alpha}\right): \alpha \in \Lambda\right\}$ is an open cover of $X$ and for every finite family $\left\{F_{i}: i \in k\right\}$ of compact subsets of $Y$ with $\bigcap_{i=1}^{k} F_{i}=\emptyset$ we have $\bigcap_{i=1}^{k} \overline{f^{-1}\left(F_{i}\right)}=\emptyset$.

The localic version of Theorem 1 is the following

Theorem 3. Let $A_{j}$ be a dense sublocale of a locale $A, B$ a regular locale, then continuous map $f: A_{j} \rightarrow B$ has a unique extension to a continuous map $g: A \rightarrow B$ if $f$ satisfies that for each family $\left\{b_{\alpha}: \alpha \in \Lambda\right\}$ with $\bigvee\left\{b_{\alpha}: \alpha \in \Lambda\right\}=1$, there exists a family $\left\{a_{i}: i \in I\right\}$ such that $\bigvee\left\{a_{i}: i \in I\right\}=1$ and $\left\{j\left(a_{i}\right): i \in I\right\}$ is a refinement of $\left\{f^{*}\left(b_{\alpha}\right): \alpha \in \Lambda\right\}$.

Corollary. (A. Joyal) Let $A_{j}$ be a flat sublocale of a locale $A, B$ a compact regular locale; then every continuous map $f: A_{j} \rightarrow B$ has a unique extension to a continuous map $g: A \rightarrow B$. (see $[7, p .91])$

Remark. Note that our proof was inspired to some extent by Joyal's proof.

Before we discuss further non-separated spaces, we need to introduce a property, which arises very naturally in algebraic geometry and in the spectral theory of rings and lattices and which partially makes up for the loss of separation. Firstly, a closed subset $A$ of a space $X$ is called irreducible, if it is not the union of two closed proper subsets. Now we say a space is sober if each nonvoid closed irreducible subset has a unique dense point. It is clear that every Hausdorff space is sober and every sober space is $T_{0}$.

For open sets $U, V$ of a space $X$ we write $V \ll U$ if for each open covering $U$ of $U$ there is some finite part of $U$ which covers $V$. Thus $V \ll U$ implies $V \subseteq U$ and $U \ll U$ implies that $U$ is quasi-compact. Following [5], we say a space $X$ is core-compact if for each $x \in X$ and $U \in O(X)$ with $x \in U$, there is some $V \in O(X)$ 
with $x \in V \ll U$. The relation $\ll$ is known as the way below relation and is one of the fundamental attributes of a continuous lattice. A full discussion of core-compactness can be found in [5]; in particular, it is noted there that a space $X$ is core-compact exactly when $O(X)$ is a continuous lattice and that a sober space $X$ is core-compact exactly when it is locally quasicompact.

Finally let us say a space $X$ is stable if for each open sets $U_{1}, U_{2}, V_{1}, V_{2}$ of $X, V_{1} \ll U_{1}$ together with $V_{2} \ll U_{2}$ implies $V_{1} \cap V_{2} \ll U_{1} \cap U_{2}$. Following [0], we say a space $X$ is well-compacted if

(i) $X$ is quasi-compact sober;

(ii) $X$ is core-compact, (equivalently, locally quasicompact);

(iii) $X$ is stable.

Clearly, every compact Hausdorff space is well-compacted.

Recall that a space is spectral if it is quasicompact sober and has a base of quasicompact open sets which is closed under finite intersections. Spectral spaces are exactly the spaces occurring as spectra of distributive lattices with 0 and 1 , or of commutative rings with 1 . In [9], it is shown that a space is well-compacted if and only if it is a retract of a spectral space; in particular, every spectral space is well-compacted.

Now we turn to the second main result in this paper. In the sequel, all space are assumed only to have $T_{0}$-separation unless otherwise specified.

To show the following theorem, we need another property. We say a dense subset $A$ of a space $X$ is flat if there is a lattice homomorphism $s: O(A) \rightarrow O(X)$ such that $s(U) \cap A=U$ for each $U \in O(A)$.

Example. Every $T_{1}$ space is a flat subset of its Wallman compactification; in particular, each discrete (normal) space is a flat subset of its Ston-Čech compactification.

Theorem 4. Let $A$ be a flat subset of a space $X$ and $f$ a continuous mapping of $A$ to $Y$, where $Y$ is well-compacted; then $f$ has a continuous extension over $X$.

Proof: As the proof of Theorem 1, we define $g^{*}: O(Y) \rightarrow O(X)$ as follows:

for each $V \in O(Y), g^{*}(V)=\bigcup\left\{s\left(f^{-1}(\operatorname{Int} Q)\right): Q \subseteq V, Q\right.$ quasicompact $\}$

where $s$ is the lattice homomorphism. We can show that $g^{*}$ preserves finite meets and arbitrary joins. In particular, we have $g^{*}(Y)=X$ and $g^{*}(\emptyset)=\emptyset$.

Now we define $g_{*}: O(X) \rightarrow O(Y)$ as follows:

$$
\text { for each } U \in O X, g_{*}(U)=\bigcup\left\{V \in O Y: g^{*}(V) \subseteq U\right\}
$$

Thus we also have, for each $U \in O(X), V \in O(Y), V \subseteq g_{*}(U)$ if and only if $g^{*}(V) \subseteq$ $U$. 
Hence we can claim that for each $x \in X, g_{*}(X \backslash \overline{\{x\}})=Y \backslash \overline{\{y\}}$ for some $y \in Y$. It suffices to observes that the set $Y \backslash g_{*}(X \backslash \overline{\{x\}})$ is an irreducible closed set and that $Y$ is sober. On the other hand, for each $x \in X$, the point $y$ with $g_{*}(X \backslash \overline{\{x\}})=Y \backslash \overline{\{y\}}$ is unique since $Y$ is sober.

So we can define a mapping $g: X \rightarrow Y$ as follows:

for each $x \in X, g(x)=y$ if and only if $g_{*}(X \backslash \overline{\{x\}})=Y \backslash \overline{\{y\}}$.

Mimicing the proof of Theorem 1, we can show that $g$.is as required.

Remark. The localic version of this theorem was essentially obtained by Johnstone [7]. But our notion of flatness is more general than the one of the spatial version of localic flatness. In fact, it suffices to note that $O(A)$ is a flat sublocale of $O(X)$ if and only if $\operatorname{Ex}: O(A) \rightarrow O(X)$ is lattice homomorphism, where $\operatorname{Ex}(V)=X \backslash \overline{A \backslash V}$, and the following lemma.

LEMMA. The following conditions are equivalent for any space $X$;

(i) $A$ is a flat subset of $X$ such that for each $x \in X$ with $x \notin \bar{F}$, where $F$ is a closed subset of $A$, there is an $U \in O(A)$ with $x \in s(U)$ and $s(U) \cap F=\emptyset$;

(ii) the mapping $\mathrm{Ex}: O(A) \rightarrow O(X)$ preserves finite unions;

(iii) for each pair $F_{1}, F_{2}$ of closed subsets of $A$, we have $\bar{F}_{1} \cap \bar{F}_{2}=\overline{F_{1} \cap F_{2}}$.

Proof: (ii) $\Leftrightarrow$ (iii) and (ii) $\Rightarrow$ (i) are clear. Now we show (i) $\Rightarrow$ (iii). Suppose that $x \in \bar{F}_{1} \cap \bar{F}_{2} \backslash \overline{F_{1} \cap F_{2}}$, where $F_{1}$ and $F_{2}$ are closed sets of $A$; then there is some $U \in O A$ with $x \in s \emptyset(U)$ and $s(U) \cap F_{1} \cap F_{2}=\emptyset$ by assumption, hence $U \cap F_{1} F_{2}=\emptyset$ and $U=\left(A \backslash F_{1}\right) \cup\left(A \backslash F_{2}\right)$. So we have $s(U)=s\left(A \backslash F_{1}\right) \cup s\left(A \backslash F_{2}\right)$ and either $x \in s\left(A \backslash F_{1}\right)$ or $x \in s\left(A \backslash F_{2}\right)$, which contracts with the assumption $x \in \bar{F}_{1} \cap \bar{F}_{2}$.

The following example shows that the additional condition in the above Lemma (i) cannot be omitted.

Example. Let $X$ be the right half of the real line $(0, \infty)$ and the topology $\theta=$ $\{[0, x) \backslash A: A$ countable whenever $x<1$ and $A$ finite whenever $x \geqslant 1\}$ then $(X, \theta)$ is $T_{1}$. Now let $Y$ be the space obtained from $X$ by adding a new point $p$ such that $X$ is open and the neighbourhoods system $\mathcal{B}(p)$ of the point $p$ is $\{\{p\} \cup[0,1) \backslash B: B \subseteq$ $X$ is finite . Thus $X$ is an open dense subset of $Y$ and $y$ is also $T_{1}$. Now we show that Ex: $O(X) \rightarrow O(Y)$ does not preserve binary unions: Let $A_{1}, A_{2}$ be two disjoint infinite countable subsets of $[0,1)$; then the sets $\left([0,1) \backslash A_{1}\right)$ and $\left([0,1) \backslash A_{2}\right)$ are open in $Y$, morever, $\operatorname{Ex}\left([0,1) \backslash A_{i}\right)=[0,1) \backslash A_{i}, i=1,2$. But $\operatorname{Ex}\left(\left([0,1) \backslash A_{1}\right) \cup\left([0,1) \backslash A_{2}\right)\right)=$ $\operatorname{Ex}[0,1)=[0,1) \cup\{p\} \neq[0,1)$. 
On the other hand, we can define a lattice homomorphism $s: O(X) \rightarrow O(Y)$ such that $s(U) \cap X=U$ for each $U \in O(X)$, that is, $X$ is a flat subset of $Y$. In fact, $s$ is defined by $s(U)=U$ is infinite, and $s(U)=U \cup\{p\}$ if $X \backslash U$ is finite. It is easy to check that $s$ is as required.

Remark. No such space is regular since the assumption that $A$ is a flat subset of $X$ implies the one that each pair $F_{1}, F_{2}$ of disjoint closed sets of $A$ have disjoint closure in $X$ which is equivalent to the one that $\mathrm{Ex}: O(A) \rightarrow O(X)$ is a lat tice homomorphism in the realm of regular spaces.

In the sequel, we shall give some applications of Theorem 4 .

The next theorem will generalise the following known result due to Wallman. 'Every continuous mapping from a $T_{1}$ space to a compact $T_{2}$ space has a unique continuous extension over its Wallman compactification'.

One might have conjectured that the uniqueness would be dependent on the $T_{2}$ separation of the image space in the above result of Wallman, as usual.

To show that the $T_{2}$-separation can be omitted, let us note that, for each $T_{1}$-space $X$, each continuous mapping $f$ from its Wallman compactification $\omega X$ to a compact $T_{2}$-space $Y$ has the following property,

$\left(^{*}\right)$ for each pair $V_{1}, V_{2} \in O Y$, there is an open set $U$ of $X$ with $f^{-1}\left(V_{1}\right) \subseteq$ $U^{*} \subseteq f^{-1}\left(V_{2}\right)$ if $V_{1} \ll V_{2}$.

To see this, it suffices to note that $V_{1} \ll V_{2} \Rightarrow f^{-1}\left(V_{1}\right) \ll f^{-1}\left(V_{2}\right)$ and that $\left\{U^{*}: U \in\right.$ $O X\}$ forms a base closed under finite unions.

TheOrem 5. Let $X$ be a $T_{1}$ space and $f: X \rightarrow Y$ a continuous mapping, where $Y$ is well-compacted; then $f$ has a unique continuous extension satisfying (*) over its Wallman compactification $\omega X$.

Proof: For each $U \in O(X)$, let $s(U)=U^{*}$; then $s$ is a lattice homomorphism. It follows from Theorem 4 that $f$ has a continuous extension $g$ over $\omega X$. Noting that, for each $V \in O(Y), g^{-1}(V)=g^{*}(V)=\bigcup\left\{\left(f^{-1}(\operatorname{Int} Q)\right)^{*}: Q \subseteq V, Q\right.$ quasicompact $\}$, we have $f^{-1}\left(V_{1}\right)^{*} \subseteq g^{-1}\left(V_{2}\right)$ whenever $V_{1} \ll V_{2}$. On the other hand, we clearly have $g^{-1}\left(V_{1}\right) \subseteq\left(f^{-1}\left(V_{1}\right)\right)^{*}$. Thus we have shown that the extension $g$ satisfies $\left({ }^{*}\right)$.

Now we prove the uniqueness. Let $X \stackrel{C}{\longrightarrow} \omega X \underset{h_{2}}{\stackrel{h_{1}}{\rightrightarrows}} Y$ such that $h_{1} i=h_{2} i$, where both $h_{1}$ and $h_{2}$ satisfy $\left({ }^{*}\right)$. If $h_{1} \neq h_{2}$, that is, $h_{1}(p) \neq h_{2}(p)$ for some $p \in \omega X$, we can assume there are $V_{1}, V_{2}, U \in O(Y)$ with $h_{1}(p) \in V_{1} \ll V_{2} \ll U$ and $h_{2}(p) \notin U$ by $Y$ being well-compacted. Furthermore, we find $W_{1}, W_{2} \in O(X)$, since both $h_{1}$ and $h_{2}$ satisfy (*), such that $p \in h_{1}^{-1}\left(V_{1}\right) \subseteq W_{1}^{*} \subseteq h_{1}^{-1}\left(V_{2}\right)$ and $h_{2}^{-1}\left(V_{2}\right) \subseteq W_{2}^{*} \subseteq h_{2}^{-1}(U)$, in particular, $p \in W_{1}^{*} \backslash W_{2}^{*}$ and $W_{1} \backslash W_{2} \neq \emptyset$. Thus we can choose some $x \in X$ with $x \in W_{1} \backslash W_{2}$, hence $x \in W_{1}^{*} \backslash W_{2}^{*} ;$ so $h_{1}(x) \in V_{2}$ and $h_{2}(x) \notin V_{2}$ which contradicts 
with $h_{1} i=h_{2} i$.

Remark. This theorem generalises the above result of Wallman.

As another interesting application, we consider the well-compactifications of $T_{0}$ spaces (it is just the second part of Stone representation theorem; it was also mentioned in $[\boldsymbol{\theta}]$.

Let $L$ be a distributive lattice with 0 and 1 and $\sigma L$ denote the prime spectra of $L$ (that is, the set of all prime ideals of $L$ with the Stone-Zariski topology). Then $L$ is a spectral space. Now let $X$ be a $T_{0}$-space and $O(X)$ be the topology of $X$. Then $O(X)$ is a distributive lattice with 0 and 1 . Let $\Sigma X=\sigma O(X)$ then $\Sigma X$ is well-compacted (indeed, a spectral space). It is easy to show that $X$ is homeomorphic to the subspace $\left\{I_{x}: x \in X\right\}$, where $I_{x}=\{U \in O(X): x \notin U\}$, of $X$, that is, the mapping $I: X \rightarrow \Sigma X$ defined by:

for each $x \in X, I(X)=I_{x}$ is continuous, relatively open and $I(X)$ is dense in $\Sigma X$.

Thus we have shown that $\Sigma X$ is a well-compactification of $X$. Now we want to establish some counterparts of Theorem 5. To do this, we define $s: O(X) \rightarrow O \Sigma X$ as follows:

$$
\text { for each } U \in O(X), \quad s(U)=\{p \in \Sigma X . U \notin p\} \text {. }
$$

Then it is easy to check that $s$ is a lattice homomorphism such that $s(U) \cap X=U$, that is, $X$ is a flat subset of $X$.

Following Simmons, we say a continuous map $f: S \rightarrow T$ is well compacted if for each open sets $U, V$ of $T$, the implication

$$
V \ll U \Rightarrow f^{-1}(V) \ll f^{-1}(U) \quad \text { holds. }
$$

As another application of our Theorem 4, we give a new proof of the following theorem essentially due to Simmons $([\boldsymbol{9}])$.

Theorem 6. Let $X$ be a $T_{0}$ space and $f: X \rightarrow Y$ a continuous mapping, where $Y$ is well compacted. Then $f$ has a unique continuous well-compacted extension over $\Sigma X$.

Proof: By virtue of Theorem 4, we have a continuous extension $g$ of $f$ such that $g^{-1}(V)=g^{*}(V)=\bigcup\left\{s f^{-1}(\operatorname{Int} Q): Q \subseteq V, Q\right.$ quasicompact $\}$. Noting each $s(U)$ is quasicompact open, so we see that $g$ is well-compacted. The proof of the uniqueness is similar to that in Theorem 5 . 


\section{REFERENCES}

[1] J. Dugundji, Topology (Allyn and Bacon, Boston, 1966).

[2] S. Eilenberg and N. Steenrod, Foundations of Algebraic Topology (Princeton University Press, 1952).

[3] R. Engelking, General Topology (PWN, Warsaw, 1977).

[4] R. Engelking, 'Remarks on real compact spaces', Fund. Math. 55 (1964), 303-308.

[5] K.H. Hofmann and J.D. Lawson, 'The spectral theory of distributive continuous lattices', Trans. Amer. Math. Soc. 248 (1978), 285-310.

[6] W.N. Hunsaker and S.A. Naimpally, 'Extensions of functions: reflective functors', Bull. Austral. Math. Soc. 35 (1987), 445-470.

[7] P.T. Johnstone, Stone Spaces (Cambridge Studies in Advanced Math. No.3, 1982).

[8] P.J. Johnstone, 'The Gleason cover of a topos', J. Pure Appl. Algebra 22 (1981), 229-247.

[ө] H. Simmons, 'A couple of triples', Topology Appl 13 (1982), 201-223.

Department of Mathematics

Shanghai Institute of Mechanical Engineering

Slianghai

Peoples Republic of China 